\title{
Short-scar facelift technique combining malar fat pad suspension and lamellar low superficial musculo-aponeurotic system excision
}

\author{
Angel A. de Larrazabal, MD, FPSCS, FACS ${ }^{1}$, Corazon Collantes-Jose, MD, FPSCS ${ }^{2}$ \\ ${ }^{1}$ Department of Cosmetic Surgery, De Los Santos Medical Center, Quezon City, Philippines, ${ }^{2}$ Corazon Aesthetics, Makati, Philippines
}

\begin{abstract}
Background: Minimally invasive surgery is already at the forefront in all surgical specialties, including cosmetic surgery. Hence, we have adapted and modified our technique to offer a short-scar facelift with minimal tissue dissection and manipulation to surgically rejuvenate the aging face.

Objective: This study aimed to surgically address the aging face and neck by employing this modified short-scar rhytidectomy with minimal dissection, manipulation, and using the correct vector suited for Asian faces as an alternative and innovative surgical procedure.

Methods: We performed this short-scar facelift by combining the malar fad pad suspension and lamellar low superficial musculoaponeurotic system (SMAS) excision

Results: From 2015 to 2017, we have performed 51 facelift procedures with our modification, with 8 men and 43 women, with a mean age of 64.30 years (range, $50-78$ years). Of the 51 patients, 29 patients (56.86\%) had regular follow-ups. Three complications were noted. One developed post-operative hematoma. The other developed seroma formation. The third developed split thickness skin necrosis. Majority of our patients were satisfied with the results.

Conclusion: This combined malar fat pad suspension and lamellar low SMAS excision technique is a simple, safe, and effective alternative procedure for facelift.

Level of Evidence: $\mathrm{V}$
\end{abstract}

Keywords: malar fat pad suspension; short-scar facelift; SMASectomy; superficial musculo-aponeurotic system excision

\section{Introduction}

The advancement in modern medicine, particularly in the field of cosmetic surgery, has provided us an in depth understanding of the facial anatomy and its important landmarks [1-4], as well as understanding the changes that occur in the aging face.

In 1995, Yousif [5] noted these characteristic changes that occur in the central third of the face associated with aging. A youthful midface is characterized by prominent cheeks and a smooth transition between the lower eyelid and cheek. Structural, soft tissue, and skin changes develop as wrinkles and creases, progressive ptosis, and general atrophy of structures.

In 2000, Pessa [6] reiterated these changes in the aging face in relation to the skin, soft tissues, and bone structures.

Different facelift procedures have been developed to surgi-

Received February 20, 2018; Revised April 16, 2018; Accepted April 16, 2018

Corresponding author: Angel A. de Larrazabal

E-mail: delarrazabalangel@yahoo.com; angeldlzmd@gmail.com, ORCID: https://orcid.org/0000-0003-3711-0564

This is an Open Access article distributed under the terms of the Creative Commons Attribution Non-Commercial License (http://creativecommons.org/licenses/by-nc/4.0), which permits unrestricted non-commercial use, distribution, and reproduction in any medium, provided the original work is properly cited.

Copyright (c) 2018 Korean Society of Korean Cosmetic Surgery and Medicine (KSKCS \& KCCS). 
cally rejuvenate the aging face, and each has its own merit [7], and interest in superficial musculo-aponeurotic system (SMAS) excision has gained popularity.

In 1997, Baker [8] introduced the lateral SMASectomy to the surgical community. He championed this alternative to the formal elevation of the superficial fascia by performing a lateral SMASectomy, which involves the removal of a portion of the SMAS in the region directly overlying the anterior edge of the parotid gland. Superficial fascia excision in this region secures the mobile anterior SMAS to the fixed portion of the superficial fascia overlying the parotid gland.

After lateral SMASectomy, the medial SMAS is pulled superolaterally following the orientation of the zygomaticus major muscle and the subsequent SMAS closure lie perpendicular to the nasolabial fold, thereby producing improvement not only of the nasolabial fold, but also of the jowl and jawline. This method represents a rapid, safe, and reproducible surgery, allowing the surgeon the versatility obtained with formal SMAS flap while producing both safety and rapidity of SMAS plication [9].

Several modifications to the lateral SMASectomy have evolved, and those of interest are the modifications introduced by Waterhouse et al. [10] in 2007 and Park et al. [11] in 2013. In contest, Marten [12] in 2008 introduced the lamellar high SMASectomy. He believed that the traditional low cheek SMAS flap elevated below the zygomatic arch has a disadvantage because it cannot, by design, exert an effect on tissues of the midface and infraorbital region. Low designs target the lower cheek and jowl only and produce little if any improvement in the upper anterior cheek and midface areas. He thought that planning the flap higher, along the superior border of the zygomatic arch, and extending the dissection medially to mobilize midface tissue, overcomes this problem and allows a combined, simultaneous lift of the jawline, cheek, and midface with a single unified flap. The outcome is improved, and no separate midface lift procedure is needed.

In 2002, De Cordier et al. [13] introduced the technique of elevating the malar fat pad to rejuvenate the midface.

In 2006, Noone [14] described an approach to midfacial rejuvenation that combines the elements of SMAS plication and lateral SMASectomy with a suture suspension of the malar fat pad to achieve a long-term improvement of the aging midface. However, in 2007, Tonnard and Verpaele [15] popularized this type of malar fat pad suspension. The minimal access cranial suspension (MACS) lift, a short-scar face lift for the lower and middle thirds of the face, uses a pure vertical vector and in- volves no lateral tension. This type of malar fat pad suspension was the third purse string suture in their extended MACS lift. The third malar suture provides an additional correction of the middle third of the face and results in the extended MACS lift [16].

Since then, procedures combining a short-scar facelift with SMAS manipulation have gained popularity over the past 15 years for patients with early signs of mid- and lower-facial laxity who seek rhytidectomy [17].

Minimally invasive surgery is already at the forefront in all surgical specialties, including cosmetic surgery. Hence, we have adapted and modified our technique to offer a short-scar facelift with minimal tissue dissection and manipulation to surgically rejuvenate the aging face.

This study aimed to surgically address the aging face and neck by employing this modified short-scar rhytidectomy with minimal dissection, manipulation, and using the correct vector suited for Asian faces as an alternative and innovative surgical procedure.

\section{Materials and methods}

The highlights of this technique involve meticulous dissection under clear and direct vision to prevent injury with regard to facial anatomy and plane of dissection.

This technique follows the standard short-scar facelift incision; however, with our own modifications. The skin incision is made starting from the temporal anterior hair line $2 \mathrm{~cm}$ above the lateral tip of the eyebrow, traversing the sideburns then along pre-auricular and pre-tragal areas, and ending just at the back of ear lobule. The facial skin flap is created with meticulous dissection after tumescent infiltration. The first part in the technique is the malar fat pad suspension. This is the third purse string suture (malar loop) devised by Drs. Patrick L. Tonnard and Alexis M. Verpaele and is used to suspend the malar fad pad. This suture addresses the mid-face rhytides.

The anchor point is on the deep temporal fascia, just lateral to the lateral orbital rim in front of the path of the frontal branch of the facial nerve. We subsequently created a window in the orbicularis muscle to the deep temporal fascia, wherein we take a deep bite, anchoring the suture to the deep temporal fascia. The purse-string suture is oriented obliquely downward and medially toward the malar fad pad. At the pre-operatively marked point $2 \mathrm{~cm}$ below the lateral canthus, we reverse the direction of suturing upward and laterally. The loop has a narrow $U$ shape and ends at its starting point. The knot is tied under maximal 
tension.

The second part in the technique is the lamellar low SMASectomy that addresses the lower face (lower cheek and jowl). We determine a point $1 \mathrm{~cm}$ anterior to the tragus. From this starting point, we subsequently draw 2 to $3 \mathrm{~cm}$ horizontal line (infrazygomatic) and 3 to $5 \mathrm{~cm}$ vertical line downward, followed by tumescent infiltration of the SMAS area before the SMAS flap is created. The SMAS flap is pulled vertically and laterally in the direction of the zygomaticus major muscle to the desired tension, and a stay suture is applied. Excess tissues are excised, and edges of the SMAS are approximated with running suture.

The facial skin is re-draped with a supero-lateral (slightly vertical) vector and under minimal tension. Excess skin is fashioned, excised, and sutured appropriately. In our clinic, the procedure may be performed under local anesthesia

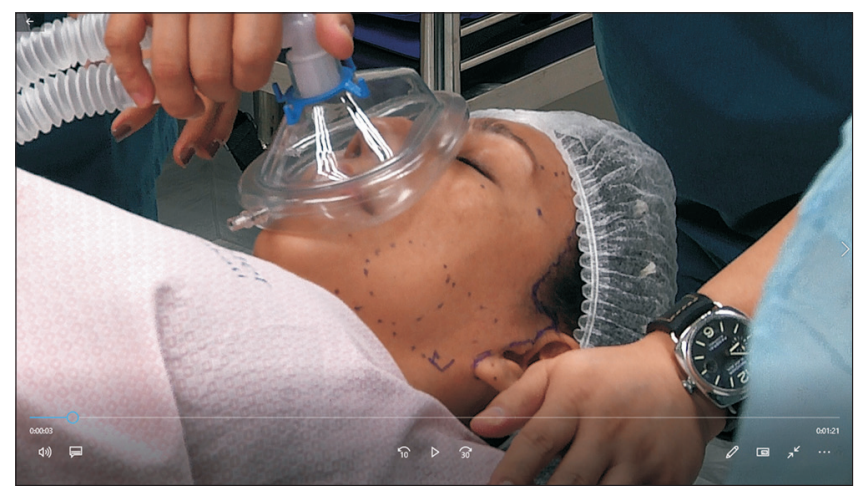

Fig. 1. Preoperative markings placed while patient is on upright positing (sitting). In the photograph, patient is already supine and administerd with anesthesia. or with intravenous sedation and can be combined with blepharoplasty, rhinoplasty, and lower face syringe lipocontouring.

There is less hematoma, bruising, and pain. Our patients are happy and satisfied and can go home right after the procedure.

\section{Operative technique}

1. Pre-operative markings are placed while the patient is on upright position (sitting).

The patient is already supine and is administered with anesthesia as shown in Fig. 1.

2. The patient is placed in supine position under IV sedation as shown in Fig. 2.

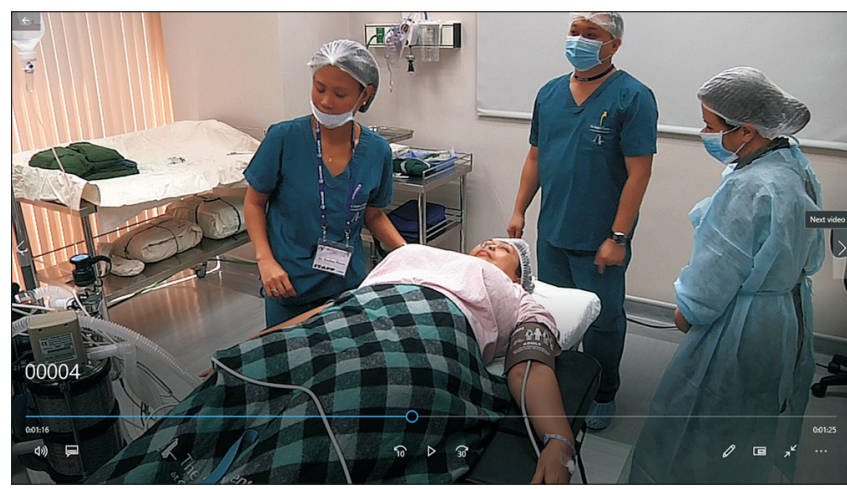

Fig. 2. Patient placed in supine position under intravenous sedation.
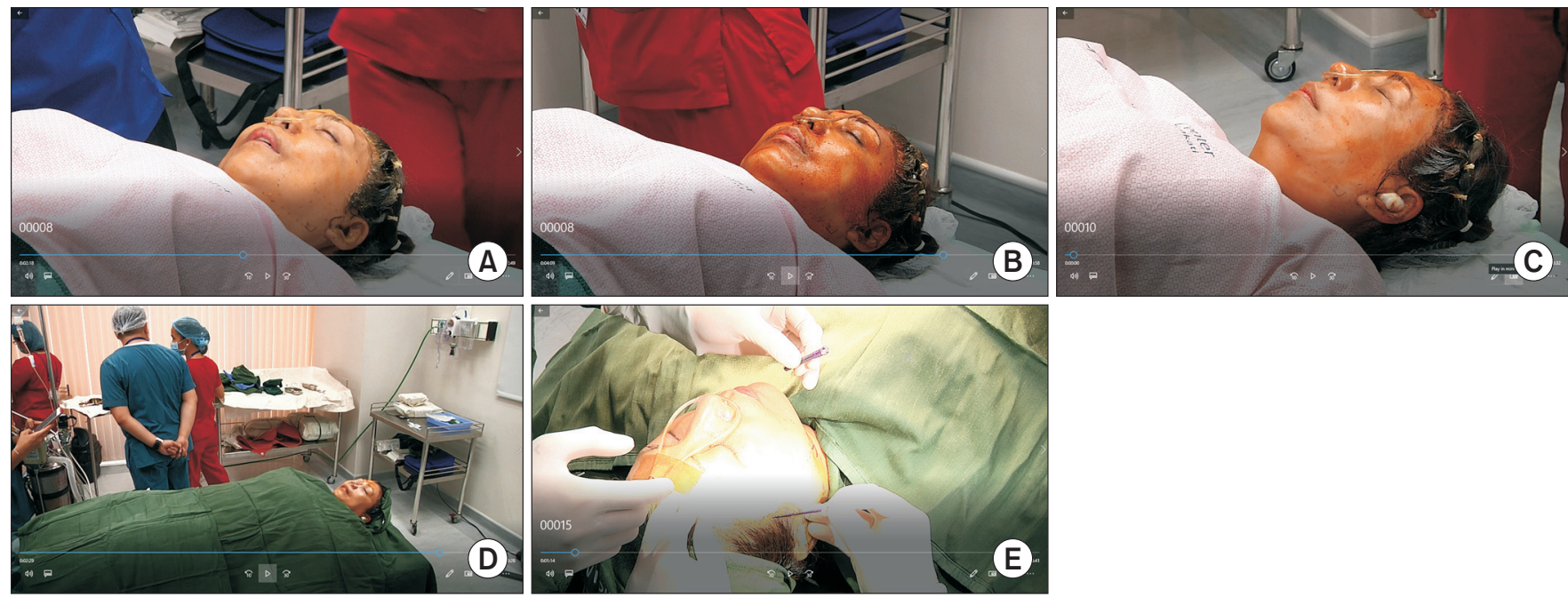

Fig. 3. Asepsis and antisepsis prep then sterile donning. (A) After betadine wash. (B) After betadine antiseptic. (C) After saline wash. (D) Patient fully donned. (E) Intra-operative markings. 
3. Asepsis and antisepsis preparation is performed, followed by sterile donning shown in Fig. 3.

3.a. After betadine wash.

3.b. After betadine antiseptic.

3.c. After saline wash.

3.d. Patient fully donned.

3.e. Intra-operative markings.

4. Infiltration of tumescent solution into the subcutaneous layer over the areas to be dissected using $1.5 \mathrm{~mm}$ infiltrating cannula (500 $\mathrm{ml}$ normal saline solution $+20 \mathrm{ml} 2 \%$ lidocaine+0.5 ml epinephrine) as shown in Fig. 4 .

4.a. Temporal and malar areas.

4.b. Lower cheek and jowls.

4.c. Sub-mandibular and sub-mental areas.

4.d. Waiting period of 30 minutes after tumescent infiltration.

5. Syringe lipo-contouring of the jowls, lower face, submental, and sub-mandibular areas with 2-3 mm suction cannula (if warranted) as shown in Fig. 5. 5.a. Jowls.

5.b. Sub-mandibular area.

5.c. Sub-mental area.

6. An incision is made over the hairline (saw-tooth and oblique) and pre-auricular area, which could be extended to the post-auricular area as shown in Fig. 6.

6.a. Local infiltration anesthesia into incision site.

6.b. Local infiltration anesthesia into incision site.

6.c. Beginning of the incision from the anterior temporal hairline.

6.d. Saw-toothed and beveled technique.

7. Sharp and blunt dissections are performed to create the skin flap and hemostasis as shown in Fig. 7.

7.a. Initial dissection is on the lower face, jowls, and submandibular areas.

7.b. Followed by the zygomatic and malar areas.

7.c. Finally, the temporal area exposing the orbicularis oculi muscles.
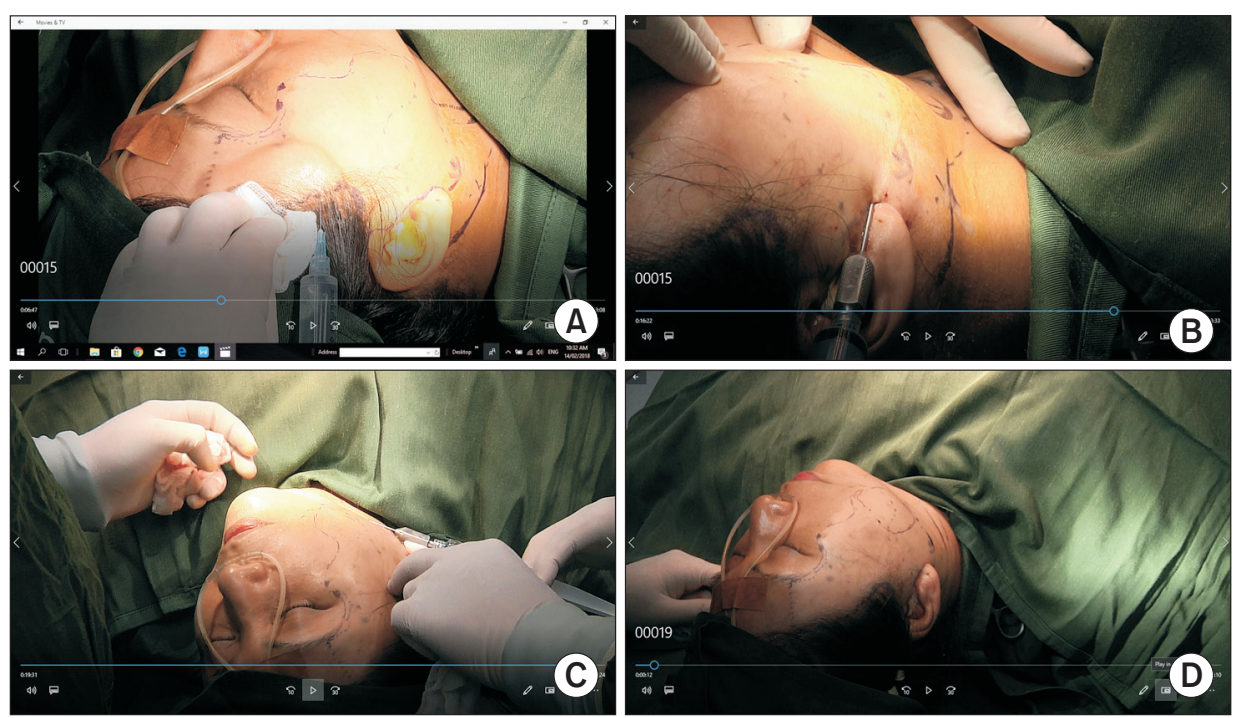

Fig. 4. Infiltration of tumescent solution into the subcutaneous layer over the areas to be dissected using $1.5 \mathrm{~mm}$ infiltrating cannula $(500 \mathrm{ml}$ normal saline solution $+20 \mathrm{ml} 2 \%$ lidocaine $+0.5 \mathrm{ml}$ epinephrine). (A) Temporal and malar areas. (B) Lower cheek and jowls. (C) Sub-mandibular and sub-mental areas. (D) Waiting period of 30 minutes after tumescent infiltration.
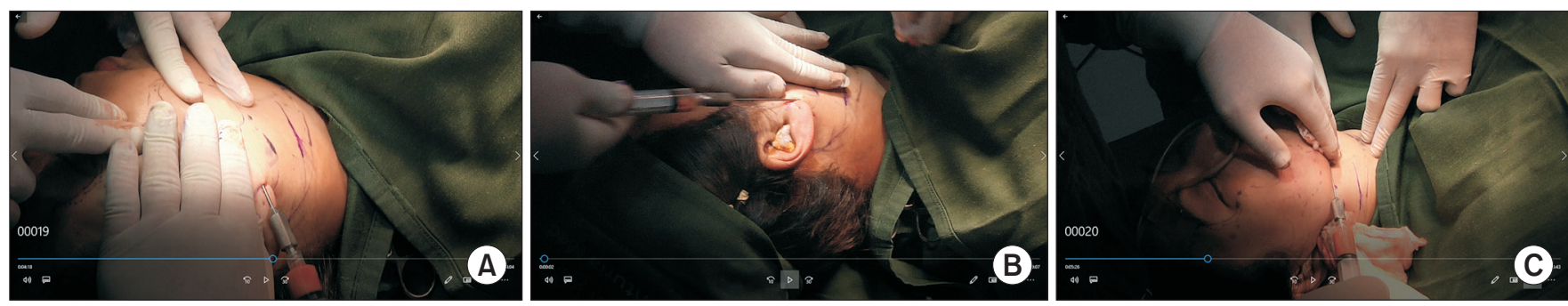

Fig. 5. Syringe lipo-contouring of the jowls, lower face, sub-mental, and sub-mandibular areas with 2-3 mm suction cannula (if warranted). (A) Jowls. (B) Sub-mandibular area. (C) Sub-mental area. 


\section{Malar fat pad anchored to the deep temporal fascia} as described using prolene 4-0 as shown in Fig. 8.

8a. Markings placed and identification of the supero-lateral border of the malar fat pad.

8.b. Demonstrating the malar fat pad suture suspension technique.

8.c. Making the knot in maximal tension.
9. SMASectomy (lamellar low) as described using prolene 4-0 as shown in Fig. 9.

9.a. Markings placed.

9.b. Tumescent infiltration into the sub-SMAS.

9.c. Incision is made on the SMAS to the correct dissection plane.

9.d. Sharp dissection of SMAS.

9.e. Showing the limit of dissection of SMAS within $3 \mathrm{~mm}$
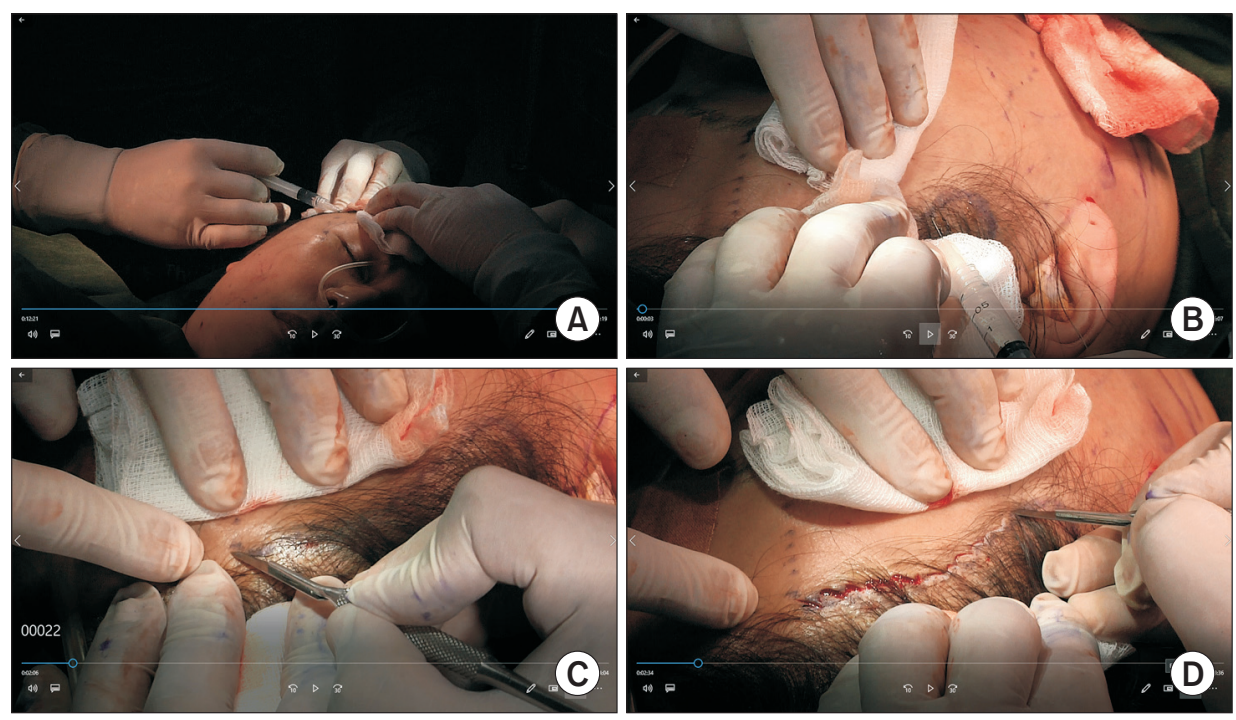

Fig. 6. Incision is made over the hairline (saw-tooth and oblique) and pre-auricular area and could be extended to the postauricular area. (A) Local infiltration anesthesia into incision site. (B) Local infiltration anesthesia into incision site. (C) Beginning of incision from the anterior temporal temporal hairline. (D) Sawtoothed and beveled technique.
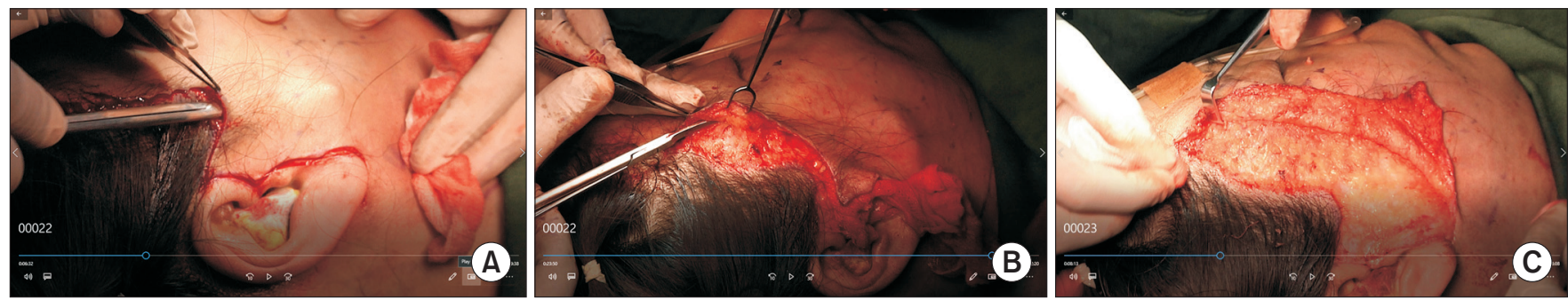

Fig. 7. Sharp and blunt dissection is done to create the skin flap; hemostasis. (A) Initial dissection is on the lower face, jowls, and submandubular areas. (B) This followed by the zygomatic and malar areas. (C) Then finally the temporal area exposing the orbicularis oculi muscles.
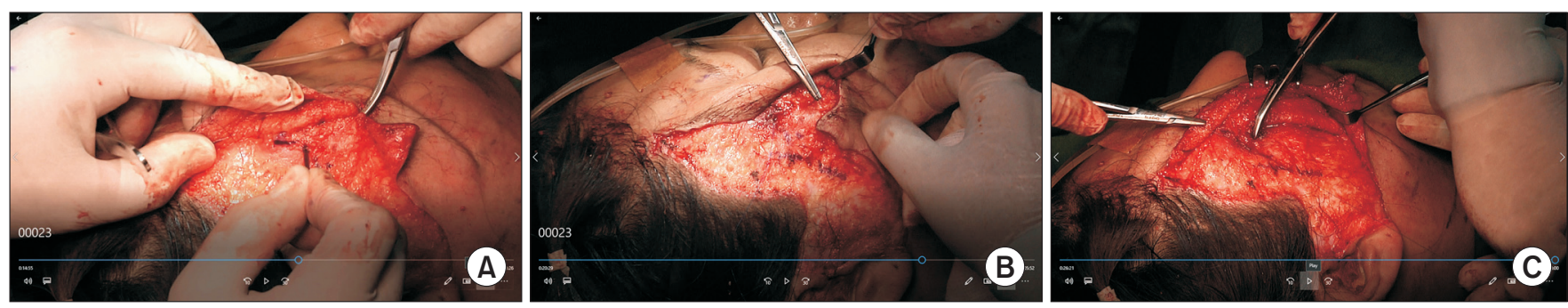

Fig. 8. Malar fat pad anchored to the deep temporal fascia as described using prolene 4-0. (A) Markings placed and identification of the supero-lateral border of the malar fat pad. (B) Demonstrating the malar fat pad suture suspension technique. (C) Making the knot in maximal tension. 
medially of the anterior border of masseter muscle.

9.f. Showing the vector of the SMAS.

9.g. Guy suture applied.

9.h. SMAS excised.

9.i. Approximation of the borders of resected SMAS with running suture.

9.j. After final closure.
10. Excision of the buccal fat (anterior direct approach if warranted).

\section{Final hemostasis.}

12. Placement and anchoring of suction drain (if warranted).
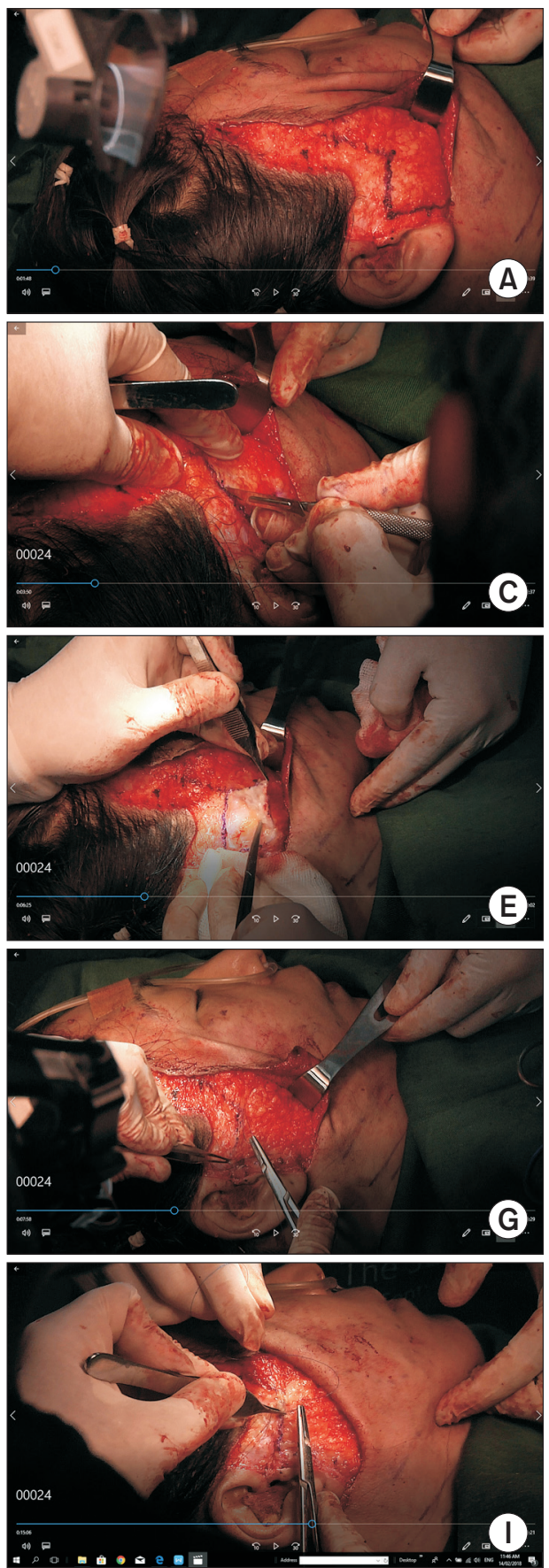
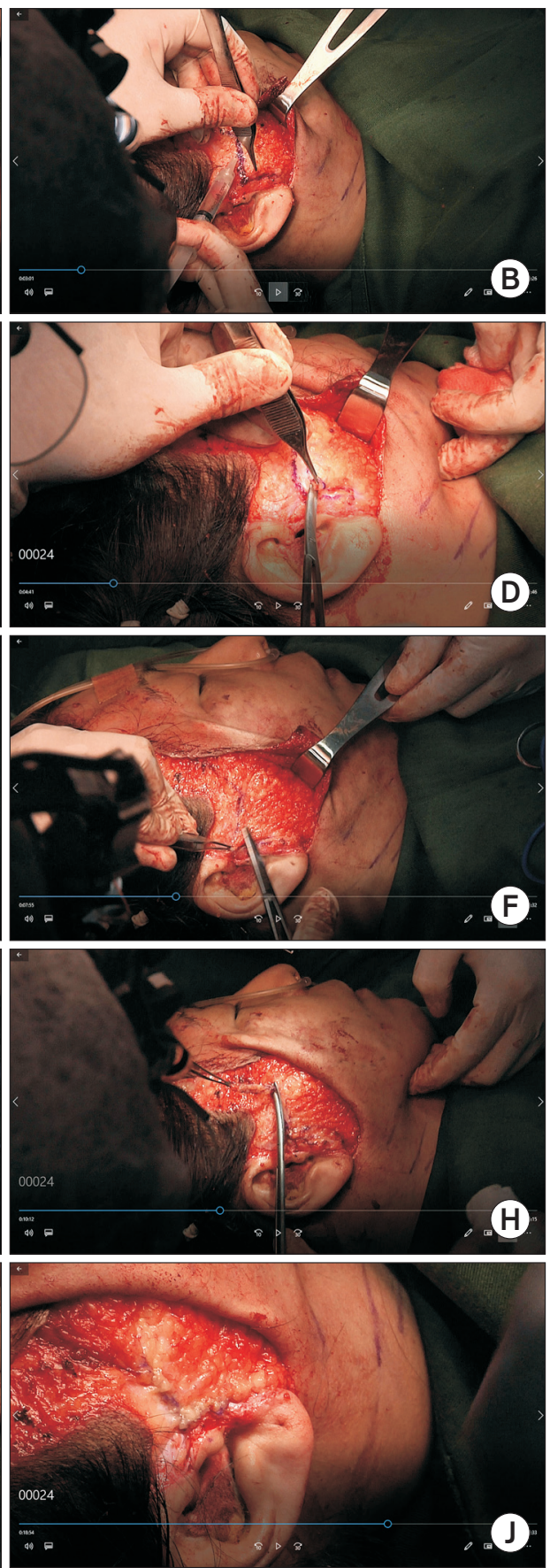

Fig. 9. SMASectomy (lamellar low) as described using prolene 4-0. (A) Markings placed. (B) Tumescent infiltration su-SMAS. (C) Incision made on the SMAS down the correct dissection plane. (D) Sharp dissection of SMAS. (E) Showing the limit of dissection of SMAS within 3 $\mathrm{mm}$ medially of the anterior border of masseter muscle. (F) Showing the vector of the SMAS. (G) Guy suture applied. (H) SMAS excised. (I) Approximation of the borders of resected SMAS with running suture. (J) After final closure. SMAS, superficial musculo-aponeurotic system. 


\section{Redraping of skin and placement of guy suture as} shown in Fig. 10.

10. Guy suture placed after the correct vector of pull of the skin is determined.

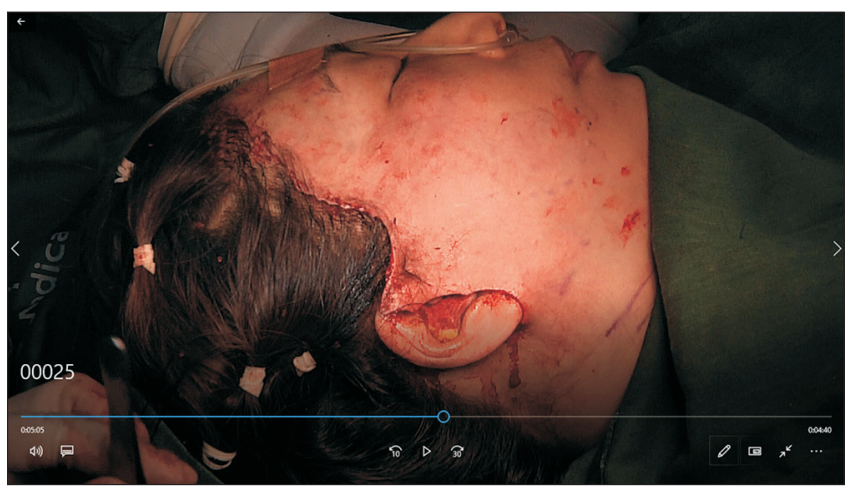

Fig. 10. Redraping of skin and placement of guy suture. Guy suture placed after the correct vector of pull of the skin has been determined.
14. Markings are placed, and excess skin is removed as shown in Fig. 11.

11.a. Markings are placed on the skin to be excised.

11.b. Fashioning and removing excess skin.

15. Sub-dermal closure using monocryl 5-0 as shown in Fig. 12.

12.a. Sub-dermal suturing at key areas.

12.b. Skin closure with nylon (not shown).

16. Skin closure using prolene 5-0 over the hairline incision wound and prolene 6-0 at the pre-auricular incision wound. The same is performed on the contralateral side.

Application of bandages (tegaderm or opsite).

\section{Results}

From 2015 to 2017, we have performed 51 facelift procedures with our modification, with 8 men and 43 women, with a mean age of 64.30 years (range, 50-78 years). Regular follow-ups were
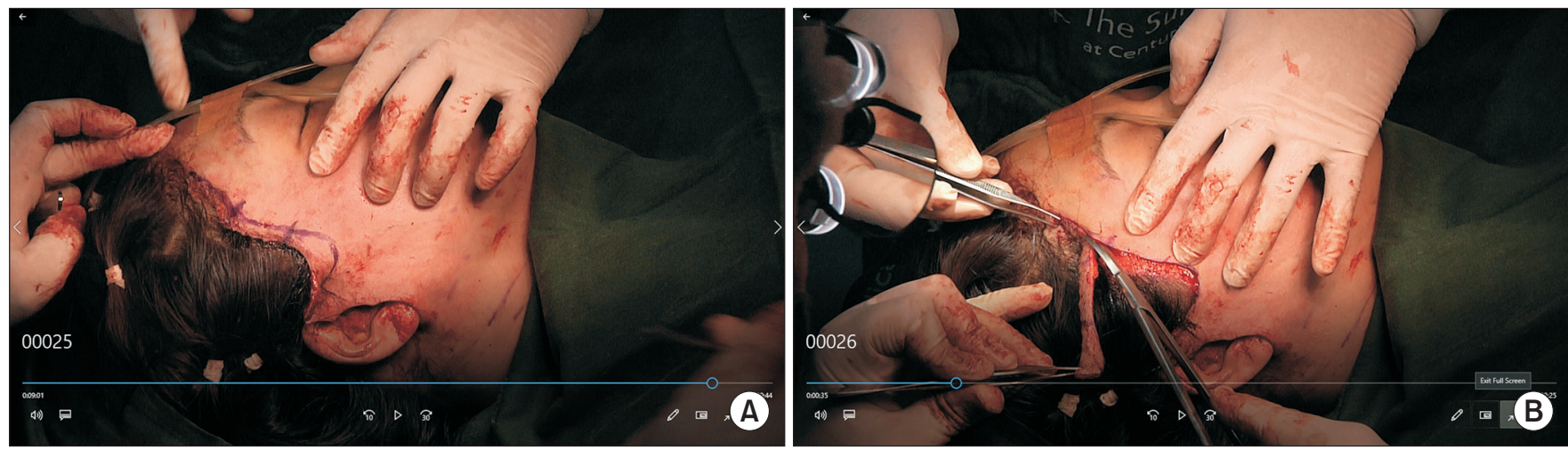

Fig. 11. Markings placed and removal of excess skin. (A) Markings placed on the skin to be excised. (B) Fashioning and removal of excess skin.
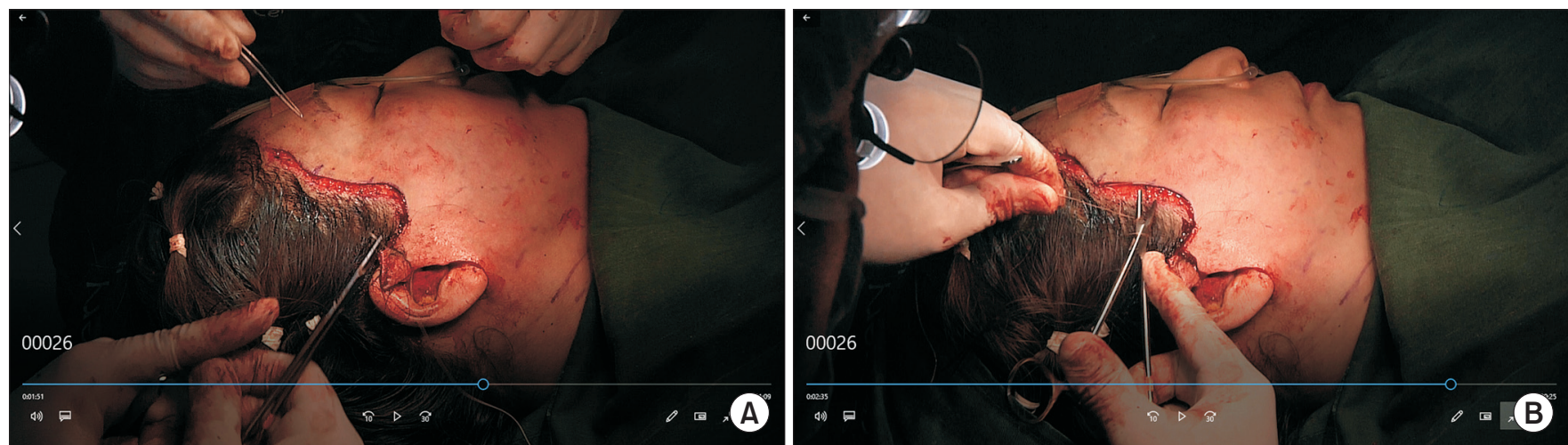

Fig. 12. Sub-dermal closure using monocryl 5-0. (A) Sub-dermal suturing at key areas. (B) This is followed by skin closure with nylon, however not shown. 
determined at 1 week, 2 weeks, 1 month, 3 months, 6 months, and 1 year. However, our patients were poorly compliant. Of the 51 patients, 29 patients (56.86\%) had regular follow-ups.

Three complications were noted. One developed post- operative hematoma that required evacuation, exploration, and control of bleeders. The other one developed seroma formation that needed aspiration and was treated conservatively. The third one developed split thickness skin necrosis, which was
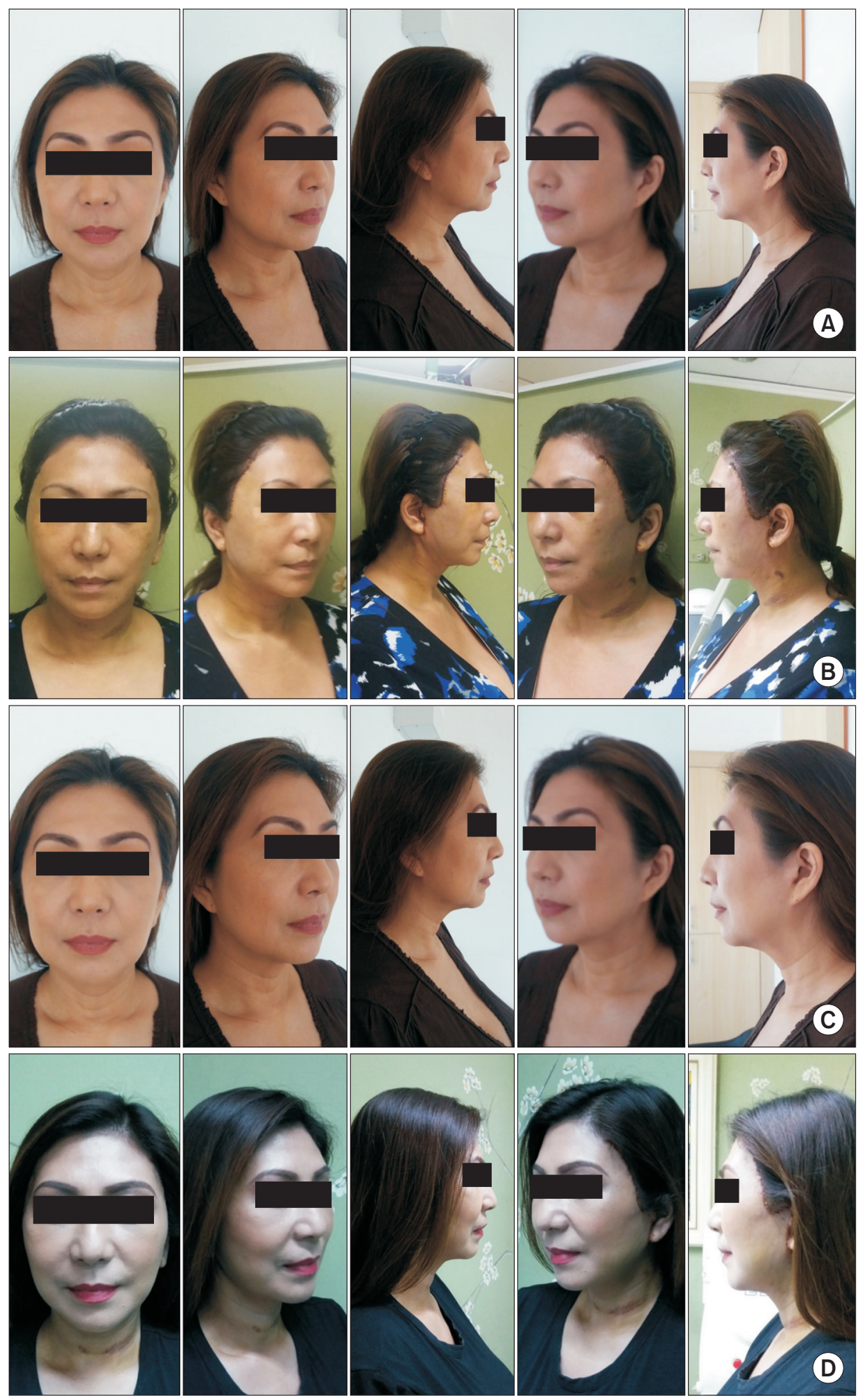

(A)

Fig. 13. This figure shows the preoperative and post-operative photographs of the same patient shown intraoperatively. (A) Preoperation. (B) One week postoperation. (C) Preoperation. (D) One month postoperation. 
managed conservatively with aquacell wound dressing.

Majority of our patients were satisfied with the results. However, no objective outcome tool was used to determine statistical significance as we only used photographs and testimonials.

Fig. 13 shows the pre-operative photographs of the same patient shown intra-operatively, and the post-oprative photographs at 1 week and at 1 month, respectively.

This 57-year-old woman was very satisfied and happy with the outcome of her procedure using our technique. Note that we were able to surgically rejuvenate her aging face with the shallowing of the naso-labial and naso-jugal folds, returning the youthful face of an inverted triangle, and elevating the lateral wing of her eyes without the tell-tale signs of facelift surgery.

\section{Discussion}

Because of the small sample size, neither statistical analysis can be performed nor any valid recommendation can be suggested. Although our patients were satisfied with the result, this is a level 5 evidence. We intend to pursue this, increase our patient population, have a more objective analysis, and make a more concrete recommendation.

In contrast, we were able to demonstrate that this technique could accomplish a simple, safe, and effective surgical rejuvenation procedure and can be considered as an alternative technique to address the aging face even with short-scar facelift incision, minimal dissection, and manipulation of soft tissues.

\section{Conflicts of interest}

The authors have nothing to disclose.

\section{References}

1. Mendelson BC, Freeman ME, Wu W, Huggins RJ. Surgical anatomy of the lower face: the premasseter space, the jowl, and the labiomandibular fold. Aesthetic Plast Surg 2008;32:185-95.

2. Rohrich RJ, Pessa JE. The retaining system of the face: histologic evaluation of the septal boundaries of the subcutaneous fat compartments. Plast Reconstr Surg 2008;121:1804-9.

3. Rohrich RJ, Ghavami A, Lemmon JA, Brown SA. The individualized component face lift: developing a systematic approach to facial rejuvenation. Plast Reconstr Surg 2009;123:1050-63.
4. Rohrich RJ, Pessa JE. The anatomy and clinical implications of perioral submuscular fat. Plast Reconstr Surg 2009;124:26671.

5. Yousif NJ. Changes of the midface with age. Clin Plast Surg 1995;22:213-26.

6. Pessa JE. An algorithm of facial aging: verification of Lambros's theory by three-dimensional stereolithography, with reference to the pathogenesis of midfacial aging, scleral show, and the lateral suborbital trough deformity. Plast Reconstr Surg 2000;106:479-88; discussion 489-90.

7. Nahai F, Nahai FR, Ford DT, Tonnard PL, Marten TJ, Stuzin JM, et al. Part V. Face lift and neck lift. In: Nahai F, editor. The art of aesthetic surgery: principles and techniques. Vol. 2. Face \& neck lift, midface rejuvenation, rhinoplasty, hair transplantation. St. Louis, MO: Quality Medical Pub.; 2005. p. 827-1357.

8. Baker DC. Lateral SMASectomy. Plast Reconstr Surg 1997; 100:509-13.

9. Baker DC. Lateral SMASectomy, plication and short scar facelifts: indications and techniques. Clin Plast Surg 2008;35:53350.

10. Waterhouse N, Vesely M, Bulstrode NW. Modified lateral SMASectomy. Plast Reconstr Surg 2007;119:1021-6; discussion 1027-8.

11. Park TH, Park JH, Whang KW. Modified lateral SMASectomy. Plast Reconstr Surg 2013;132:880e-1e.

12. Marten TJ. High SMAS facelift: combined single flap lifting of the jawline, cheek, and midface. Clin Plast Surg 2008;35:569603.

13. De Cordier BC, de la Torre JI, Al-Hakeem MS, Rosenberg LZ, Costa-Ferreira A, Gardner PM, et al. Rejuvenation of the midface by elevating the malar fat pad: review of technique, cases, and complications. Plast Reconstr Surg 2002;110:152636; discussion 1537-40.

14. Noone RB. Suture suspension malarplasty with SMAS plication and modified SMASectomy: a simplified approach to midface lifting. Plast Reconstr Surg 2006;117:792-803.

15. Tonnard P, Verpaele A. The MACS-lift short scar rhytidectomy. Aesthet Surg J 2007;27:188-98.

16. Verpaele A, Tonnard P, Gaia S, Guerao FP, Pirayesh A. The third suture in MACS-lifting: making midface-lifting simple and safe. J Plast Reconstr Aesthet Surg 2007;60:1287-95.

17. Vincent D. Short scar rhytidectomy techniques. Atlas Oral Maxillofac Surg Clin North Am 2014;22:37-52. 